\title{
Quale operatore, per quale venipuntura, per quale paziente in emodialisi?
}

\author{
Marco Lombardi ${ }^{1}$, Giuseppe Quintaliani ${ }^{2}$ \\ ${ }^{1} \mathrm{SS}$ di Emodialisi del Mugello, Azienda Sanitaria di Firenze, Firenze \\ ${ }^{2} \mathrm{SC}$ Nefrologia, Dialisi e Trapianto, Perugia
}

\begin{abstract}
WHICH OPERATOR, WHICH VENIPUNCTURE TECHNIQUE, AND FOR WHICH HEMODIALYSIS PATIENT?
Abstract. The buttonhole technique (BT) does not represent an alternative venipuncture technique, but can be described as another distinct venipuncture technique for arteriovenous fistula. This introduction to the issue will try to examine all BT criticalities, especially pointing out the crucial role of a good organization when facing these criticalities.
\end{abstract}

Key words: Buttonhole technique, Criticalities, Coordination, Skills, Clinical competence

Conflict of interest: None.

Financial support: None.

Accettato: 26 Marzo 2014

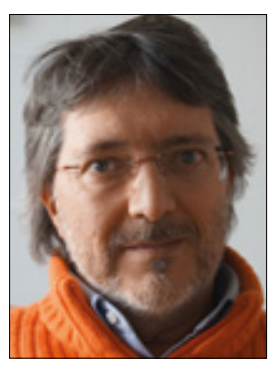

"Quando vorrete vi proverò l'esistenza di Dio al contrario..." [Louis-Ferdinand Céline. Pantomima per un'altra volta. Einaudi Ed.]

Lombardi Marco

Credo sia difficile smentirci quando affermiamo che la tecnica di venipuntura a sito costante o Buttonhole Technique (BT) non è una tecnica di venipuntura alternativa, bensì un'altra tecnica di venipuntura dell'accesso vascolare per emodialisi. In effetti, come per tutto ciò che viene applicato ai pazienti, anche questa tecnica ha le sue criticità, che devono essere analizzate e capite sia per le cause che le possono indurre sia, e soprattutto, per vedere se sia possibile minimizzarle o, ancora meglio, evitarle.

La controversia sulla BT che apre questo numero del Giornale, scaturita da un articolo di Marcello Napoli (1), è stata pensata coinvolgendo indubbi cultori della materia, per offrire al lettore la possibilità di farsi un'idea "propria" sullo stato dell'arte della BT. In questa breve "intro" alla controversia, infatti, abbiamo cercato di appaiare l'esperienza personale di un metodologo e quella di un gestore di un centro dialisi alle dotte dissertazioni degli Autori coinvolti, non tanto per trarre delle conclusioni ma per dare modo al lettore di riflettere, ponderare ed eventualmente applicare. Tutto, ovviamente, parte dal, e arriva al, paziente, passando, altrettanto ovviamente, per l'Organizzazione. Cercheremo di spiegarci.

Come ben esprime Decenzio Bonucchi, tutti vorremmo un paziente partecipe e coinvolto nelle scelte cliniche e, in particolare, nella gestione del proprio accesso vascolare. L'Organizzazione non solo fa capolino, ma diventa "essenziale", quando si afferma che la puntura dell'accesso vascolare dovrebbe essere sempre una manovra ben strutturata, personalizzata e monitorata: per fare ciò, dovrebbe essere sempre adottato un "piano di utilizzo" della FAV, piano che dovrebbe essere originato dalla condivisione delle scelte più appropriate dello staff e dalle caratteristiche psico-fisiche del paziente. Ancora, sul piano organizzativo, sono da citare le possibili carenze strutturali, capaci di ostacolare la collaborazione tra figure sanitarie a cui, sempre organizzativamente, si può associare (come riportato da Galli, Zollo e Cavatorta) l'ancora più deleteria possibile abitudine di ritenere la venipuntura dell'accesso vascolare una ...banale manovra amanuense... sino ad arrivare al possibile rifiuto "prevenuto" verso il nuovo o il cambiamento.

Ma il nodo centrale, a nostro avviso, sta nel rapporto tra la cosiddetta "skill" degli anglosassoni, ovvero la capacità di chi è chiamato a praticare la tecnica, e l'Organizzazione.

Non siamo, ovviamente, tutti ugualmente capaci in tutto! E, proprio per "regolare o normalizzare" questa imprescindibile possibile differenza è d'obbligo considerare l'Organizzazione: qualcuno dovrà pur essere preposto a decidere di mantenere o meno in quella data funzione chi è abile o non abile a svolgerla!!! E, quindi, non possiamo non citare ancora Galli 
et al., che riportano che ...la puntura nella BT non può essere affidata a un qualsiasi puntore, magari dopo un affrettato addestramento, e magari non sufficientemente abile nella puntura con l'ago smusso. La BT, infatti, non è una semplice variante della puntura a sito variabile, ma, al contrario, è una tecnica di incannulamento del vaso del tutto differente e, pertanto, da affidare solo a personale idoneo e motivato, tenendo presente che la manualità con gli aghi smussi viene acquisita con la pratica e la costanza.

L'Organizzazione non è, però, quella che deve/può soltanto mantenere o allontanare chi di dovere; no, l'Organizzazione è, secondo noi, preposta preventivamente e continuativamente a preparare, addestrare e aggiornare ogni figura professionale nella sanità. Ancora, Galli et al. sono chiari quando riportano l'esperienza di Imperia ...dove la BT è stata iniziata nel Giugno del 2006 ed è stata progressivamente estesa negli anni seguenti alla maggior parte dei pazienti con buoni risultati, in particolare senza complicanze infettive e con un alto gradimento da parte del personale e dei pazienti. Tuttavia, negli ultimi 2 anni, 2012 e 2013, si sono verificati alcuni casi di gravi complicanze infettive da stafilococco aureo (endocardite e spondilodiscite), il tutto in concomitanza con un elevato turnover del personale infermieristico e con una riorganizzazione dirigenziale e la conseguente perdita della figura medica di riferimento per la BT ...l'estensione della tecnica a tutto il personale di assistenza, l'elevata rotazione del personale con lo spostamento di infermieri particolarmente addestrati e abili nel loro lavoro, l'aumento probabilmente eccessivo del numero dei pazienti trasferiti a questa tecnica, il ritmo di lavoro "stressante", specialmente nella fase di attacco e stacco, simile, nei grandi centri dialisi, alla catena di montaggio delle fabbriche, e la progressiva perdita di attenzione alle procedure legata alla ripetitività della manovra... sono stati il nucleo delle criticità di questa tecnica in un grosso centro che crede nella stessa. Anche nel ben più piccolo centro dove lavora uno dei due Autori di questa introduzione, dopo 3 anni senza complicanze con la BT, in concomitanza con la riduzione del personale infermieristico e, quindi, per sopperire a una carenza insostenibile, con l'ingresso di personale nuovo e meno esperto associato al rientro di personale dopo una lunga inattività e, quindi, meno "allenato", sono comparse le prime e numerose complicanze della tecnica. L'Organizzazione è centrale non solo a Imperia ma ovunque perché ...la BT non è una semplice variante della puntura a scala di corda, ma è una tecnica del tutto diversa, al punto da richiedere non solo una riorganizzazione del lavoro e dei compiti dello staff, ma, soprattutto, un vero e proprio cambiamento culturale che dovrebbe riguardare non solo il personale addetto (quello in dialisi) ma anche la Dirigenza Medico-Infermieristica, la Direzione Sanitaria e l'Organizzazione Sindacale... ma, soprattutto, dovrebbe volere ...un numero limitato di pungitori in grado di praticare la puntura non solo con l'ago tagliente ma anche con l'ago smusso... (Galli, Zollo e Cavatorta).

Altri Autori, altre località, ma stesse esperienze, stessi concetti: Monica Spina scrive che ...nella nostra esperienza, $i$ risultati migliori si sono avuti nei pazienti che si auto pungono e in quelli che dializzano nel CAD di Serramanna. Infatti, nel CAD, il turnover degli infermieri è inferiore e il cari- co di lavoro è meno gravoso rispetto a quello dell'U.O. di San Gavino; questo ha permesso di dedicare più attenzione alla tecnica BT e di estenderla a quasi tutti i pazienti che vi si dializzano. L'acquisizione di una tecnica di venipuntura che viene effettuata sempre dalla stessa mano con il tempo si perfeziona e annulla le complicanze...; Francesco Bruni riferisce di essere riuscito a ovviare alle problematiche, focalizzando gli sforzi sulle ...difficoltà organizzative, soprattutto nel primo periodo, per far coincidere $i$ turni degli operatori con quelli dei pazienti che iniziavano la metodi$c a . .$. e acquisendo maggiori tempi per il posizionamento degli aghi; Luigi Tazza et al. enfatizzano che ...cura particolare è richiesta nelle prime 2-3 settimane di BT per la formazione e il consolidamento del canale venocutaneo. Sono possibili varie tipologie di organizzazione del lavoro infermieristico: tutte richiedono profonda consapevolezza, unità di intenti, notevole spirito di collaborazione e buona volontà. Certamente, non tutti i centri potranno contare su un pool di esperti operatori disposti a sincronizzare i propri turni lavorativi con quelli del paziente prescelto per la BT, allo scopo di lasciare la creazione del canale venocutaneo nelle mani di un'unica persona, fino al momento in cui ogni infermiere potrà procedere all'applicazione degli aghi smussi...; infine, Marisa Pegoraro afferma che ...la BT non ha in sé delle criticità, ma che le stesse sono superabili con una "buona pratica clinica". Come tutte le buone pratiche necessita di attenzione, approccio strutturato e momenti di verifica e di riadattamento delle procedure. Per questo, è necessaria la creazione di ruoli di competenza avanzata come il Referente degli Accessi Vascolari... ma ci preme segnalare che, sulla stessa lunghezza d'onda, è il lavoro di Marina Sgreccia et al., che trova posto nelle pagine a seguire sempre di questo numero ampiamente dedicato alla BT.

In conclusione, anche se alla comunità scientifica mancano degli studi ben disegnati che speriamo arrivino quanto prima, allo stato attuale dell'arte crediamo di poter affermare che la BT sia una diversa tecnica di venipuntura della FAV, che può essere afflitta da problematiche di non poco peso (infezioni), la cui soluzione potrà essere trovata, tanto per cambiare, nel fattore umano, un fattore che dipende ampiamente da come è "Organizzato". Infatti, se la vera differenza sta in chi è chiamato ad applicare la tecnica, l'Organizzazione è non tanto quell'entità preposta a scegliere infermieri di "serie A" piuttosto che di "serie B", ma, piuttosto, è quell'entità chiamata a formare e a mantenere con una skill appropriata (... ai pazienti!) il personale che opera al suo interno e a fornire risorse in tutti i sensi affinché tutto ciò possa essere reale.

Insomma, il punto centrale, come nella professione medica, sta divenendo sempre più la "clinical competence". La SIN aveva avviato un ragionamento sulla "clinical competence" del nefrologo, che è fatta di skill, di volume di attività (2), di mantenimento della stessa competenza, di esperienza e di valutazione dei risultati. E non è certo da sottovalutare che, all'interno delle "core competence" del nefrologo, ASN abbia inserito il core curriculum degli accessi vascolari (3), segno che l'accesso vascolare e il percorso di "clinical competence" sono considerati core business e non certo residuali all'interno di un centro dialisi. Anche in ambito infermieristico, si parla 
di competenza nel momento in cui si accenna al "Case Manager" in ambito di accessi vascolari. A lui dovrebbe essere demandato il compito di istruire e controllare le capacità e la decisione su quando un infermiere può iniziare a pungere e, soprattutto, la valutazione dei risultati. La scaletta è sempre la stessa: all'interno di conoscenze, skill e attitudini, bisogna prima conoscere, poi conoscere come, poi dimostrare di saper fare e, da ultimo, fare. L'Audit professionale è il punto più alto e fondamentale della valutazione professionale di qualità e l'esperienza di Imperia, dove è stato possibile mettere in relazione infezioni e turnover degli infermieri, dovrebbe insegnare a tutti che: a) è possibile valutare e si può valutare solo ciò che si è misurato e b) alla valutazione deve seguire un'adeguata azione correttiva che deve andare verso l'alto (le direzioni infermieristiche e sanitarie) e verso il basso, rinforzando i livelli di training e di autorizzazione progressiva al lavoro. I coordinatori infermieristici hanno strumenti per fare e per incoraggiare; i meccanismi di premio basati su un punteggio da assegnare a fine anno sono un ottimo sistema per ottenere buoni risultati in termini di applicazione e fattività; è necessario, ovviamente, renderne edotti gli infermieri a inizio anno e condividere scelte e strategie, per arrivare a valutazioni oggettive e trasparenti. A questo punto non è certo pleonastico citare il bel lavoro di Maurizio Catino sul Rischio Clinico, apparso sul GIN nel 2009 (4),nell'ambito della serie su rischio clinico e che riporta una frase -assai più tranquilla dell'incipit di apertura:

... Gli operatori non sono tanto i responsabili di un incidente, quanto gli 'eredi' di difetti presenti nel sistema e generati da attori e unità organizzative distanti nel tempo e nello spazio. Quando accade un incidente in un'organizzazione complessa è l'organizzazione stessa che fallisce, e non soltanto l'individuo a più stretto contatto con l'evento stesso. Tale frase si dovrebbe applicare anche nel caso del BT ove l'individuo siamo tutti noi medici ed infermieri e l'Organizzazione resta però quella di cui facciamo tutti parte ed alla quale dovremmo da un lato dare di più e dall'altro pretendere ugualmente.

\section{Riassunto}

La Buttonhole Technique (BT) non è una tecnica di venipuntura alternativa, bensì un'altra tecnica di venipuntura dell'accesso vascolare per emodialisi. In questa introduzione alla controversia che appare su questo numero del Giornale, gli Autori cercano di analizzare quali siano le criticità di questa tecnica, evidenziando l'importanza che risiede nell’Organizzazione per far fronte alle stesse.

Parole chiave: Buttonhole Technique, Criticità, Organizzazione, Skill, Clinical Competence

Dichiarazione di conflitto di interessi: Gli Autori dichiarano di non avere conflitto di interessi.

Contributi economici degli autori: Gli Autori dichiarano di non aver ricevuto sponsorizzazioni economiche per la preparazione dell'articolo.
Indirizzo degli Autori:

Dr. Marco Lombardi

SS di Emodialisi del Mugello

Azienda Sanitaria di Firenze

Via della Resistenza

50032 Borgo San Lorenzo (FI)

lombardim@tin.it

\section{Bibliografia}

1. Napoli M. Il buttonhole: come trasformare una fistola in un catetere. Giornale di Tecniche Nefrologiche \& Dialitiche 2013; 25 (4): 290-2.

2. Quintaliani G, Cappelli G, Meneghel G. La Clinical Competence prodotta da: SIN, BEST PRACTICE, Comitato Governo Clinico Nephromeet, SIN http://www.nephromeet.com/web/
procedure/protocollo.cfm?List=WsIdEvento,WsPageNameCa 1ler,WsIdRisposta,WsRelease \&c1 $=00052 \& \mathrm{c} 2=\% 2 \mathrm{Fweb} \% 2 \mathrm{Fe}$ venti\%2FNEPHROMEET\%2Findex\%2Ecfm\&c3=4\&c4=1.

3. Maya ID, Allon M. Vascular access: core curriculum 2008. Am J Kidney Dis 2008; 51 (4): 702-8.

4. Catino M. Oltre l'errore umano. Per una teoria organizzativa degli incidenti nelle Organizzazioni. G Ital Nefrol 2009; 1: 110-7. 